Article

\title{
Association between Brachial-Ankle Pulse Wave Velocity and Microalbuminuria and to Predict the Risk for the Development of Microalbuminuria Using Brachial-Ankle Pulse Wave Velocity Measurement in Type 2 Diabetes Mellitus Patients
}

\author{
Byong-Kyu Kim ${ }^{1,+}$, Dilaram Acharya ${ }^{2,3,+} \mathbb{D}$, Deuk-Young Nah ${ }^{1, * \mathbb{D}}$, Moo-Yong Rhee ${ }^{4}$, \\ Seok-Ju Yoo ${ }^{2}$ (D) and Kwan Lee ${ }^{2}$ (D) \\ 1 Cardiology Division, Department of Internal Medicine, Gyeongju Hospital, College of Medicine, \\ Dongguk University, 87 Dongdaero, Gyeongju, Gyeongbuk 38067, Korea; bleumatin@dongguk.ac.kr \\ 2 Department of Preventive Medicine, College of Medicine, Dongguk University, Gyeongju 38066, Korea; \\ dilaramacharya123@gmail.com (D.A.); medhippo@hanmail.net (S.-J.Y.); kwaniya@dongguk.ac.kr (K.L.) \\ 3 Department of Community Medicine, Kathmandu University, Devdaha Medical College and Research \\ Institute, Rupandehi 32907, Nepal \\ 4 Cardiovascular Center, Dongguk University Ilsan Hospital, 27, Dongguk-ro, Ilsandong-gu, Goyang-si, \\ Gyeonggi-do 10326, Korea; mooyong.rhee@dumc.or.kr \\ * Correspondence: ptca@dongguk.ac.kr \\ + These authors contributed equally as joint first author.
}

Received: 8 August 2019; Accepted: 20 September 2019; Published: 26 September 2019

\begin{abstract}
Brachial-ankle pulse wave velocity (baPWV) provides a useful means of assessing cardiovascular events and diabetic complications. However, the nature of associations between baPWV and microalbuminuria (MAU) and its presence in Type 2 diabetes mellitus (Type 2 DM) have rarely been investigated. This study aimed to examine the association between baPWV and MAU coupled with prediction of MAU using baPWV measurement among Type 2 DM patients. In this cross-sectional study, we enrolled 424 Type 2 DM patients who visited the cardiology and endocrinology department at a tertiary level health care facility, Republic of Korea between 1 January 2006 to 31 December 2008. Clinical and laboratory data were collected, and risk factors associated with MAU and prediction of risk for the development of MAU using baPWV measurement. The association between MAU and baPWV was examined using multivariable logistic regression analysis and predicted MAU by using receiver operating characteristic (ROC) curve analysis. Of the 424 Type 2 DM patients, 93 (21.9\%) had MAU (20-200 $\mu \mathrm{g} / \mathrm{min})$. baPWV (cm/sec) was found to be significantly correlated with MAU levels (ug/min) $(r=0.791, p<0.001)$. Further, baPWV was significantly associated MAU with higher odds ratio (adjusted odds ratio (AOR) 10.899; 95\% confidence interval (CI) (4.518-26.292)). Similarly, smoking (AOR 5.736; 95\% CI (1.036-31.755)), and low-density lipoprotein (LDL)-cholesterol (mg/dL) (AOR 1.017; 95\% CI (1.001-1.033)) were also significantly associated with MAU. The appropriate cut-off value for baPWV to predict MAU $20 \mu \mathrm{g} / \mathrm{min}$ in our study was $1700 \mathrm{~cm} / \mathrm{sec}$ (area under ROC curve $=0.976$ ). This study shows that baPWV, cigarette smoking, and LDL-cholesterol are associated with MAU in Type $2 \mathrm{DM}$ patients and suggests that a baPWV cut-off of $1700 \mathrm{~cm} / \mathrm{sec}$ could be used to predict the presence of MAU $(20 \mu \mathrm{g} / \mathrm{min})$ in Type 2 DM patients in the Korean community.
\end{abstract}

Keywords: diabetes mellitus; microalbuminuria; pulse wave velocity 


\section{Introduction}

Diabetes mellitus is a global public health problem. In 2016, an estimated 1.6 million deaths were caused by diabetes and the number affected is predicted to increase to 552 million by 2030 [1,2]. In addition to increasing morbidity and mortality, Type 2 diabetes mellitus (Type $2 \mathrm{DM}$ ) is considered to be an independent risk factor of many cardiovascular illnesses, such as coronary artery disease, and peripheral artery disease [3-5]. On the other hand, microalbuminuria (MAU) is a clinical condition that can lead to proteinuria and cause endothelial dysfunction and atherosclerosis, and these conditions can increase arterial stiffness in Type $2 \mathrm{DM}[6,7]$. MAU has been reported to be positively associated with increased risks of cardiovascular morbidity and mortality independently of conventional cardiovascular risk factors in Type 2 DM [8]. Interestingly, MAU has also been reported to be an important risk factor for the development of cardiovascular diseases and for increased mortality among the general population, and even at borderline level of microalbumin in general populations are reported to be significantly increased risk of death [9]. However, the pathogenic mechanism of MAU is not well understood. One theory is that insulin resistance is common among patients with diabetes or dyslipidemia and results from endothelial dysfunction triggered by metabolic processes [10-12], while another posits that generalized vascular dysfunction due to arterial stiffness may result in MAU $[13,14]$.

Arterial stiffness has been reported to be an important marker of vascular damage and a risk factor of cardiovascular diseases [10,12] and albuminuria [15], and increased pulse wave velocity $(\mathrm{PWV})$ is known to be an indicator of arterial stiffness. Furthermore, a straightforward method of measuring brachial-ankle pulse wave velocity (baPWV) is now available, although it should be added that carotid-femoral pulse wave velocity (cfPWV) is the established standard method for measuring PWV [16]. However, there has been documented evidence of close correlation between baPWV and cfPWV, with similar determinants. As such, baPWV measurement may be used as an alternative to cfPWV measurement in order to investigate arterial stiffness for assessing cardiovascular risk [17]. baPWV reflects the stiffness of the aorta and peripheral arteries in lower and upper extremities, and is more applicable in general practice than cfPWV, as it uses a separate cuff for each limb, is automated, and easier to perform [18-20].

Several authors have concluded baPWV provides a useful means of quantitatively assessing cardiovascular events and diabetic complications [21-23], and cross-sectional and longitudinal studies have shown baPWV is independently associated with MAU [24-26]. Others have suggested baPWV cut-off values for the prediction of mortality [18] and the development of cardiovascular illnesses [27]. However, few Korean studies have addressed the nature of the association between MAU and baPWV in Type $2 \mathrm{DM}$ and the influences of other risk factors on this association, but did not examine the ability of baPWV to predict the presence of MAU in Type $2 \mathrm{DM}$. Although MAU can easily be performed in clinic, we hypothesized that an elevated baPWV, if found positively associated with MAU, may be a good indicator for microalbuminuria, and also it could be a useful screening tool to predict cardiovascular complications among Type 2 DM patients. Given this background, the current study was conducted to examine the association between baPWV and MAU coupled with prediction of MAU using baPWV measurement among Type 2 DM patients.

\section{Materials and Methods}

\subsection{Study Subjects}

Four hundred and twenty-four consecutive Type 2 DM patients who visited the department of cardiology and endocrinology of Dongguk University, Gyeongju Hospital from 1 January 2006 to 31 December 2008 were enrolled in this cross-sectional study. All subjects underwent baPWV and MAU testing. Patients with renal insufficiency (serum creatinine $\geq 1.5 \mathrm{mg} / \mathrm{dl}$ ) or clinical albuminuria $(\geq 200 \mu \mathrm{g} / \mathrm{min}$ ) were excluded. The 424 study subjects were allocated to one of two groups based on microalbumin level, that is, to a normoalbuminuria (NAU) group $(<20 \mu \mathrm{g} / \mathrm{min}(n=331))$ or a microalbuminuria (MAU) group $(20-200 \mu \mathrm{g} / \mathrm{min}(n=93))$, based on available literature [28]. 


\subsection{Measurement of Pulse Wave Velocity}

baPWV was measured in a quiet room controlled at $22 \pm 1{ }^{\circ} \mathrm{C}$ in an overnight fasted state. All study subjects were asked to refrain from caffeine, alcohol, and smoking during the $12 \mathrm{~h}$ period before testing. After having a 15 min rest, baPWV was measured using an automated device (VP-1000; Colin, Co. Ltd, Komaki, Japan) in the supine position [29]. Briefly, pressure waveforms of brachial and tibial arteries were obtained using occluding monitoring cuffs placed around upper arms and lower legs. Times taken for pulse waves to travel from lower legs to upper arms were recorded; distances between sampling points of baPWV were calculated automatically from subject heights. One technician performed all measurements.

\subsection{Ethics}

The study protocol was approved by the institutional review board of Dongguk University Gyeongju Hospital (approval number: 110757-201712-HR-03-01). Subject privacy, confidentiality, and anonymity were fully maintained throughout the study. A written informed consent was taken from each of the study participants after giving information about study details.

\subsection{Physical and Laboratory Measurements}

Physical measurements, such as heights and weights, were measured on the same days as baPWV tests. Similarly, hypertensions, hypercholesterolemia, smoking status, and diabetes duration were obtained at these times. Lipid profiles, which included total cholesterol, triglyceride, low-density lipoprotein cholesterol, high-density lipoprotein cholesterol, blood sugar level, hemoglobin A1C, and uric acid, were measured enzymatically. All blood samples were obtained in the morning in an overnight fasted state. Urinary microalbumin concentrations were measured using a radioimmunoassay (Dream r-10 counter, Shinjin Medics, Seoul, South Korea) according to the manufacturer's instructions.

\subsection{Statistical Analysis}

Demographic and clinical characteristics are presented as means and standard deviations (SDs) or as numbers and percentages, and were compared using the independent t-test or the Pearson's chi-square test depending on data type. Pearson's correlation analysis was used to identify the relationship between baPWV and the presence of MAU. All variables with a $p$-value of $\leq 0.1$ were entered the multivariable logistic regression analysis with backward elimination. Adjusted odds ratios and $95 \%$ confidence intervals (CIs) were calculated to determine the independence of the association between MAU and baPWV. $p$-values $<0.05$ were deemed statistically significant, and the analysis was conducted using SPSS version 20 (SPSS, Chicago, IL, USA). In addition, receiver operating characteristic (ROC) analysis in MedCalc Statistical Software version 17.9.7 was used to determine the optimal baPWV cut-off for predicting the presence of MAU [30].

\section{Results}

\section{Baseline and Clinical Characteristics of the Study Subjects}

The baseline characteristics and laboratory findings of the study subjects are summarized in Tables 1 and 2. Of the 424 Type 2 DM patients, 93 (21.9\%) had microalbuminuria. Study subjects were allocated to a normoalbuminuria (NAU) group $(n=331,<20 \mu \mathrm{g} / \mathrm{min}$ ) or a microalbuminuria (MAU) group $(n=93,20-199 \mu \mathrm{g} / \mathrm{min})$. Smoking $(14.2 \%$ vs. $26.9 \%, p=0.004)$, duration of diabetes $(7.5 \pm 5.7 \mathrm{vs}$. $10.4 \pm 8.8$ years, $p=0.001)$, and weight $(63.7 \pm 11.3 \mathrm{vs}$. $66.6 \pm 12.8 \mathrm{~kg}, p=0.036)$ were significantly different in the NAU and MAU groups (Table 1). Similarly, blood urea nitrogen (BUN) (16.3 $\pm 5.1 \mathrm{vs.}$ $19.3 \pm 8.8 \mathrm{mg} / \mathrm{dl}, p=0.002)$, microalbumin $(6.5 \pm 4.8 \mathrm{vs} .66 .9 \pm 43.0 \mathrm{ug} / \mathrm{min}, p=0.001)$, and baPWV $(1,459.15 \pm 200.44$ vs. $1,981.74 \pm 171.47 \mathrm{~cm} / \mathrm{sec}, p=0.001)$ were also significantly different in the NAU and MAU groups (Table 2). 
Table 1. Baseline clinical characteristics of the study subjects.

\begin{tabular}{llll}
\hline Variables & Normoalbuminuria $(\boldsymbol{n = 3 3 1 )} \mathbf{( \% )}$ & Microalbuminuria $(\boldsymbol{n}=\mathbf{9 3}) \mathbf{( \% )}$ & $\boldsymbol{p}$-Value \\
\hline Male, $\mathrm{n}(\%)$ & $173(52.3)$ & $57(61.3)$ & 0.077 \\
Age, years & $58.2 \pm 12.2$ & $59.5 \pm 13.0$ & 0.369 \\
HTN, n (\%) & $124(37.5)$ & $37(38.7)$ & 0.385 \\
H-Chol, n (\%) & $141(42.6)$ & $36(38.7)$ & 0.291 \\
Smoking, n (\%) & $47(14.2)$ & $25(26.9)$ & 0.004 \\
DM duration, Years & $7.5 \pm 5.7$ & $10.4 \pm 8.8$ & 0.001 \\
Height (cm) & $161 \pm 9$ & $163 \pm 10$ & 0.073 \\
Weight (kg) & $63.7 \pm 11.3$ & $66.6 \pm 12.8$ & 0.036 \\
BMI & $24.5 \pm 3.9$ & $24.8 \pm 3.3$ & 0.389 \\
SBP, mmHg & $125 \pm 16$ & $125 \pm 18$ & 0.956 \\
DBP, mmHg & $77 \pm 10$ & $76 \pm 11$ & 0.522 \\
PP, mmHg & $47 \pm 10$ & $48 \pm 11$ & 0.53 \\
\hline
\end{tabular}

HTN, hypertension; H-Chol, hypercholesterolemia; DM, diabetes mellitus; cm, centimeter; kg, kilogram, BMI; body mass index; SBP, systolic blood pressure; DBP, diastolic blood pressure; $\mathrm{PP}$, pulse pressure.

Table 2. Clinical characteristics of the study subjects.

\begin{tabular}{llll}
\hline Variables & Normoalbuminuria $(\boldsymbol{n}=\mathbf{3 3 1}) \mathbf{( \% )}$ & Microalbuminuria $(\boldsymbol{n}=\mathbf{9 3}) \mathbf{( \% )}$ & $\boldsymbol{p}$-Value \\
\hline FBS $(\mathrm{mg} / \mathrm{dl})$ & $136 \pm 54$ & $138 \pm 48$ & 0.722 \\
HbA1c $(\%)$ & $7.7 \pm 2.0$ & $8.1 \pm 2.1$ & 0.107 \\
BUN $(\mathrm{mg} / \mathrm{dL})$ & $16.3 \pm 5.1$ & $19.3 \pm 8.8$ & 0.002 \\
Creatinine (mg/dL) & $0.99 \pm 0.18$ & $1.14 \pm 0.72$ & 0.05 \\
Total cholesterol (mg/dL) & $179 \pm 41$ & $184 \pm 45$ & 0.369 \\
Triglyceride $(\mathrm{mg} / \mathrm{dL})$ & $160 \pm 113$ & $158 \pm 78$ & 0.864 \\
HDL-cholesterol (mg/dL) & $46.3 \pm 13.1$ & $45.4 \pm 12.7$ & 0.551 \\
LDL-cholesterol (mg/dL) & $103 \pm 33$ & $109 \pm 40$ & 0.138 \\
Uric Acid & $4.9 \pm 1.4$ & $5.2 \pm 1.8$ & 0.154 \\
Microalbumin $(\mu \mathrm{g} / \mathrm{min})$ & $6.5 \pm 4.8$ & $66.9 \pm 43.0$ & 0.001 \\
baPWV $(\mathrm{cm} / \mathrm{sec})$ & $1459.15 \pm 200.44$ & $1981.74 \pm 171.47$ & 0.001 \\
\hline
\end{tabular}

FBS, fasting blood sugar; HbA1c, hemoglobin A1c; BUN, blood urea nitrogen; HDL, high-density lipoprotein; LDL, low-density lipoprotein; baPWV, brachial-ankle pulse wave velocity.

In all study subjects, baPWV $(\mathrm{cm} / \mathrm{sec})$ was significantly correlated with microalbumin levels (ug/min) $(r=0.791, p<0.001)$ (Figure 1).

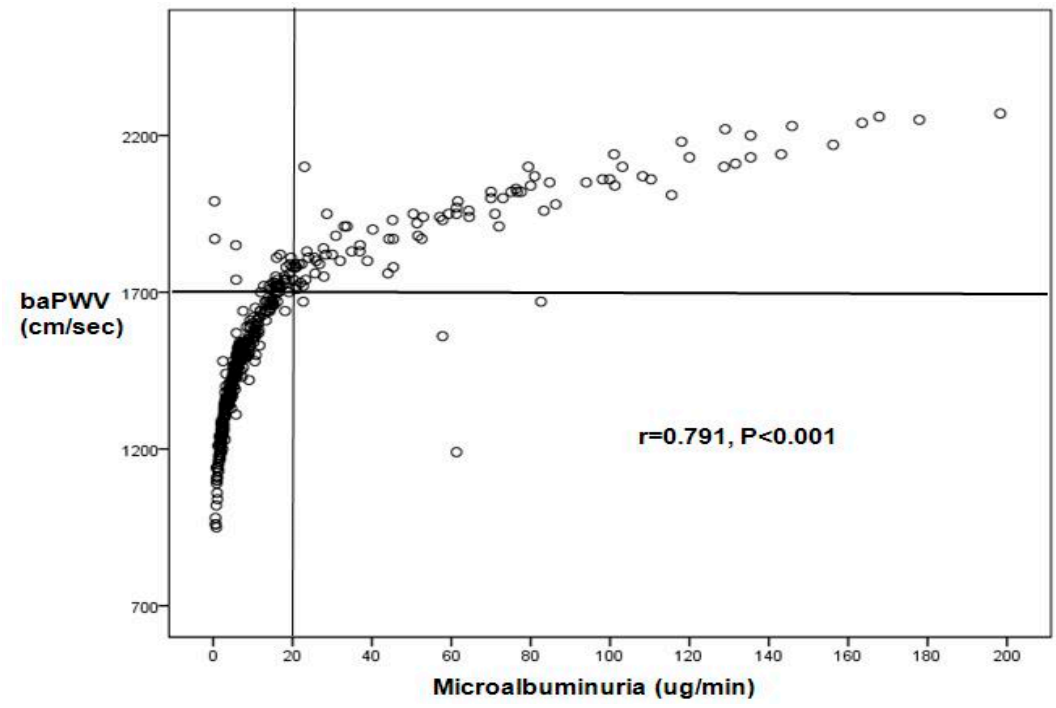

Figure 1. The relationship between microalbuminuria (MAU) and brachial-ankle pulse wave velocity (baPWV). 
Multivariable logistic regression analysis results with adjusted odds ratios and $95 \%$ confidence intervals (CIs) are shown in Table 3. baPWV was significantly associated with the MAU with higher odds ratio (AOR 10.899; 95\% CI (4.518-26.292)), and, similarly, smokers were more likely (AOR 5.736; 95\% CI (1.036-31.755)) to have MAU than nonsmokers, and those with a high LDL-cholesterol (mg/dL) were also found to be more likely (AOR 1.017; 95\% CI (1.001-1.033)) to have MAU.

Table 3. Association between brachial-ankle pulse wave velocity and microalbuminuria as determined by multivariable logistic regression analysis **.

\begin{tabular}{llllll}
\hline Variable & Regression Coefficient & SE & AOR & $\mathbf{9 5 \%}$ CI & $p$-Value \\
\hline baPWV* & 2.389 & 0.449 & 10.899 & $4.518-26.292$ & 0.0001 \\
Smoking & - & & & & \\
No & 1.747 & - & Ref [1] & - & - \\
Yes & 1.909 & 0.873 & 5.736 & $1.036-31.755$ & 0.045 \\
Creatinine $(\mathrm{mg} / \mathrm{dl})^{*}$ & 0.017 & 0.984 & 6.745 & $0.980-46.432$ & 0.052 \\
LDL-cholesterol $(\mathrm{mg} / \mathrm{dl}) *$ & 0.008 & 1.017 & $1.001-1.033$ & 0.035 \\
\hline
\end{tabular}

baPWV, brachial-ankle pulse wave velocity; SE, standard error; AOR, adjusted odds ratio; $\mathrm{CI}$, confidence interval; Ref, reference. * continuous variable. ** Adjusted for gender, smoking, diabetes mellitus duration, height, weight, blood urea nitrogen, creatinine, low-density lipoprotein, uric acid, microalbuminuria, and baPWV.

The receiver operating characteristic (ROC) curve analysis revealed that the optimum baPWV cut-off for predicting the presence of MAU $(20 \mu \mathrm{g} / \mathrm{min})$ was $1700 \mathrm{~cm} / \mathrm{sec}(95.7 \%$ sensitivity, $89.7 \%$ specificity, and area under the curve $($ AUC $)=0.976)$ ) (Figure 2).

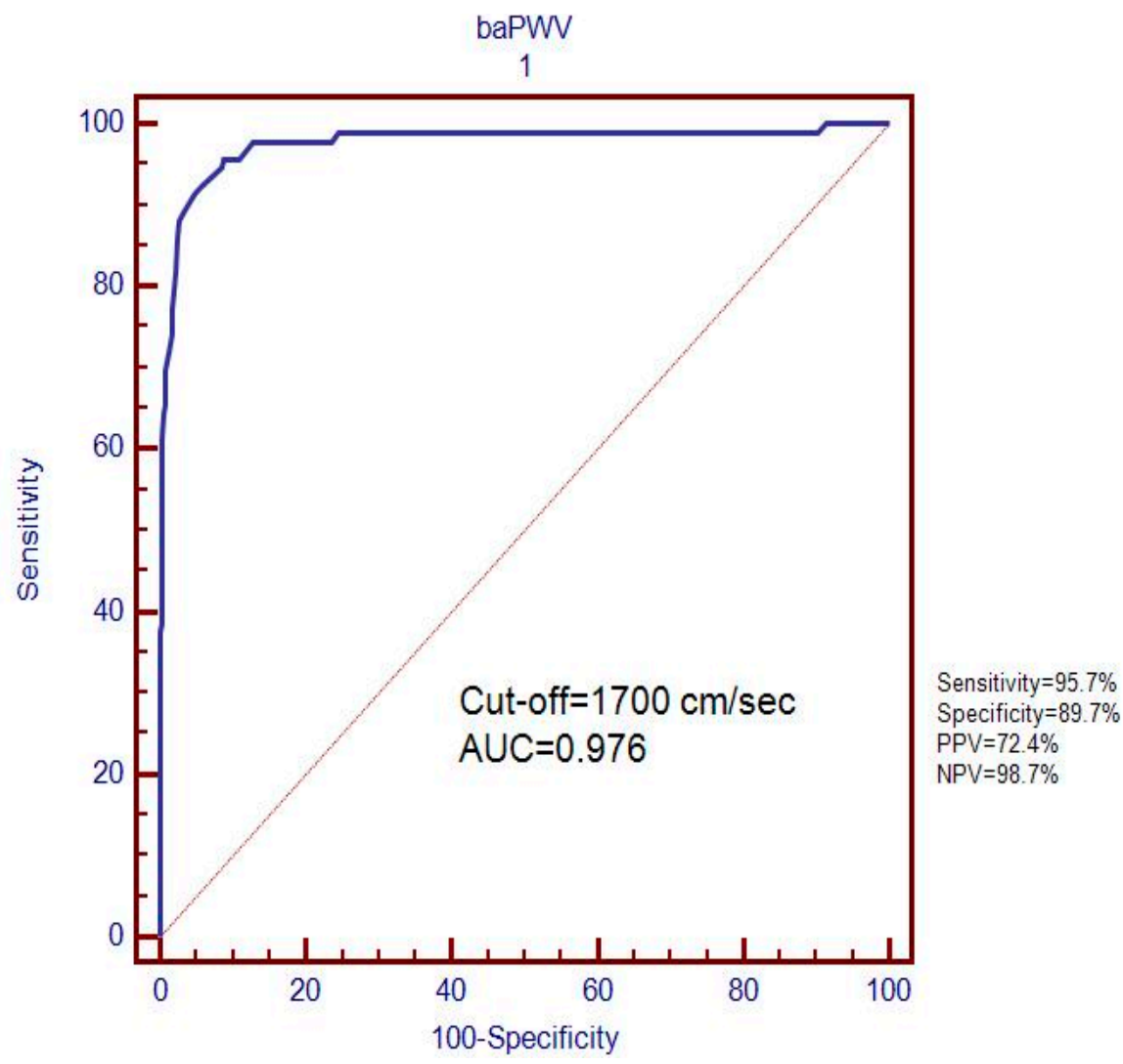

Figure 2. Determination of the optimum baPWV value for predicting the presence of microalbuminuria $(20 \mu \mathrm{g} / \mathrm{min})$.

\section{Discussion}

The principal finding of the present study was that brachial-ankle pulse wave velocity was significantly positively associated with microalbuminuria (MAU) in Type 2 DM. In addition, smoking 
and a high LDL-cholesterol were also significantly positively associated with MAU. More importantly, receiver operating characteristic (ROC) curve analysis revealed that the optimum baPWV cut-off for predicting the presence of MAU $(20 \mu \mathrm{g} / \mathrm{min})$ in Type $2 \mathrm{DM}$ was $1700 \mathrm{~cm} / \mathrm{sec}$.

This study showed that $21.9 \%$ of the 424 Type 2 DM patients included had MAU, which is much higher than the rates found in previous Korean studies [26,31]. However, previous Korean studies were conducted on healthy subjects. In addition, univariate and bivariate analyses showed that baPWV and MAU were significantly positively associated in our cohort, which is in-line with the findings of previous studies conducted in China, Japan, and South Korea [25,26,32,33]. Furthermore, this significant association remained consistent with a high odds ratio even after controlling for potential confounders. Although not precisely determined, endothelial damage is probably a major cause of MAU [10,34]. For instance, MAU becomes fixed when progression of vascular structural changes starts including glomerulosclerosis, and endothelial dysfunction could lead to the development of MAU [10], which supports the notion that MAU reflects vascular damage in diabetic patients [35,36]. Interestingly, in a large community-based Japanese study, MAU was found to be independently associated with baPWV in the general population [25], and the results obtained suggested that the general population might have been suffering from MAU.

Since MAU is linked to kidney damage and chronic kidney disease, it may be an important marker of renal and cardiovascular illnesses [37-39]. Thus, the detection of MAU among Type 2 DM patients might provide a straightforward means of identifying patients at risk and of facilitating early treatment. Kawai et al. [27] reported that a baPWV cut-off of $1750 \mathrm{~cm} / \mathrm{sec}$ might be useful for predicting the presence of cardiovascular disease and stroke in hypertensive patients, and Wang et al. suggested that a combination of baPWV $>1400 \mathrm{~cm} / \mathrm{sec}$ and a retinal artery atherosclerosis $\geq 2$ might usefully predict the presence of coronary artery disease [40]. Although these studies provided useful information regarding the detection of cardiovascular illnesses using baPWV measurements, they did not address the predictive value of baPWV for MAU in Type $2 \mathrm{DM}$. Nonetheless, despite inter-study differences, our results are in-line with previous reports. In contrast to previous studies $[21,22,26,32,41]$, we used receiver operating characteristic (ROC) curve analysis to identify an optimal baPWV cut-off for the detection of MAU (20 $\mu \mathrm{g} / \mathrm{min})$, which was found to be $1700 \mathrm{~cm} / \mathrm{sec}(95.7 \%$ sensitivity, $89.7 \%$ specificity, and AUC 0.976).

We also observed conventional risk factors of cardiovascular and renal illnesses, such as cigarette smoking and higher LDL-cholesterol, were significantly associated with MAU. Cigarette smoking has been consistently reported to be associated with MAU in longitudinal and cross-sectional studies [42,43], and, likewise, elevated levels of higher LDL-cholesterol have been reported to be associated with MAU [44]. It has been demonstrated that smoking alters glomerular filtration rate and increases urinary albumin:creatinine ratio among Type $2 \mathrm{DM}$ patients [45]. On the other hand, dyslipidemia is associated with the atherosclerotic process, and recent research has shown total cholesterol independently predicts coronary artery calcification and incidental albuminuria [46-48].

Despite its usefulness, we acknowledge that the present study has some important limitations. First, its cross-sectional nature and relatively small sample size prevent us from determining cause and effect relationships. Second, our study finding whereby baPWV cut-off of $1700 \mathrm{~cm} / \mathrm{sec}$ predicted the presence of MAU in Type $2 \mathrm{DM}$ patients in a Korean community may be not be applicable to other races. Third, our study lacks some important information on some variables, such as different cardiovascular diagnoses and correlation between cfpWV and baPWV. Fourth, study subjects were receiving hypoglycemic and antihypertensive medication, and this may have influenced our findings. Nevertheless, the association found between baPWV and MAU might indicate incipient nephropathy or cardiovascular illness in some Type $2 \mathrm{DM}$ patients [6,7], and that it might be beneficial to bring this segment of the population in for appropriate medical intervention when a simple and useful measurement is known. 


\section{Conclusions}

In the present study, baPWV, cigarette smoking, and a high LDL-cholesterol level were found to be associated with MAU among Type 2 DM patients. In addition, the study indicates a baPWV value of $1700 \mathrm{~cm} / \mathrm{sec}$ might be an important cut-off value for predicting the presence of MAU $(20 \mu \mathrm{g} / \mathrm{min})$ in Type $2 \mathrm{DM}$ patients in the Korean community. Further, longitudinal, large-scale, multicenter studies are required to investigate the association between baPWV and microalbuminuria, pulse wave velocity (PWV), carotid intima-media thickness (IMT), and ambulatory blood pressure (ABP) in Korean Type 2 DM patients.

Author Contributions: B.-K.K., D.-Y.N. and M.-Y.R. conceptualized the study and performed investigations. D.A. analyzed data with the help of K.L. and S.-J.Y., B.-K.K., and D.A. wrote the first draft of the manuscript with significant contribution and supervision from D.-Y.N., M.-Y.R., S.-J.Y. and K.L. All authors involved in writing the manuscript, editing, providing critical comments, and subsequent revisions. All authors approved the final version of the manuscript.

Funding: This research received no external funding.

Conflicts of Interest: The authors have no conflicts of interest to declare.

\section{References}

1. Whiting, D.R.; Guariguata, L.; Weil, C.; Shaw, J. IDF diabetes atlas: Global estimates of the prevalence of diabetes for 2011 and 2030. Diabetes Res. Clin. Pract. 2011, 94, 311-321. [CrossRef]

2. World Health Organization. Diabetes: Key Facts. Available online: https:/www.who.int/news-room/factsheets/detail/diabetes (accessed on 9 September 2019).

3. Juutilainen, A.; Lehto, S.; Ronnemaa, T.; Pyorala, K.; Laakso, M. Type 2 diabetes as a "coronary heart disease equivalent": An 18-year prospective population-based study in Finnish subjects. Diabetes Care 2005, 28, 2901-2907. [CrossRef] [PubMed]

4. Selvin, E.; Erlinger, T.P. Prevalence of and risk factors for peripheral arterial disease in the United States: Results from the National Health and Nutrition Examination Survey, 1999-2000. Circulation 2004, 110, 738-743. [CrossRef] [PubMed]

5. Yusuf, S.; Hawken, S.; Ounpuu, S.; Dans, T.; Avezum, A.; Lanas, F.; McQueen, M.; Budaj, A.; Pais, P.; Varigos, J.; et al. Effect of potentially modifiable risk factors associated with myocardial infarction in 52 countries (the INTERHEART study): Case-control study. Lancet 2004, 364, 937-952. [CrossRef]

6. Mogensen, C.E. Microalbuminuria predicts clinical proteinuria and early mortality in maturity-onset diabetes. N. Engl. J. Med. 1984, 310, 356-360. [CrossRef] [PubMed]

7. Naidoo, D.P. The link between microalbuminuria, endothelial dysfunction and cardiovascular disease in diabetes. Cardiovasc. J. S. Afr. 2002, 13, 194-199. [PubMed]

8. Mattock, M.B.; Morrish, N.J.; Viberti, G.; Keen, H.; Fitzgerald, A.P.; Jackson, G. Prospective study of microalbuminuria as predictor of mortality in NIDDM. Diabetes 1992, 41, 736-741. [CrossRef] [PubMed]

9. Arnlov, J.; Evans, J.C.; Meigs, J.B.; Wang, T.J.; Fox, C.S.; Levy, D.; Benjamin, E.J.; D’Agostino, R.B.; Vasan, R.S. Low-grade albuminuria and incidence of cardiovascular disease events in nonhypertensive and nondiabetic individuals: The Framingham Heart Study. Circulation 2005, 112, 969-975. [CrossRef] [PubMed]

10. Abdelhafiz, A.H.; Ahmed, S.; El Nahas, M. Microalbuminuria: Marker or maker of cardiovascular disease. Nephron Exp. Nephrol. 2011, 119, 10. [CrossRef] [PubMed]

11. Dabla, P.K. Renal function in diabetic nephropathy. World J. Diabetes 2010, 1, 48-56. [CrossRef] [PubMed]

12. Nah, D.Y.; Lee, C.G.; Bae, J.H.; Chung, J.W.; Rhee, M.Y.; Kim, J.H.; Kim, Y.S.; Kim, Y.K.; Lee, M.M. Subclinical renal insufficiency range of estimated glomerular filtration rate and microalbuminuria are independently associated with increased arterial stiffness in never treated hypertensives. Korean Circ. J. 2013, 43, 255-260. [CrossRef] [PubMed]

13. Lin, C.Y.; Chen, M.F.; Lin, L.Y.; Liau, C.S.; Lee, Y.T.; Su, T.C. Insulin resistance is the major determinant for microalbuminuria in severe hypertriglyceridemia: Implication for high-risk stratification. Intern. Med. 2008, 47, 1091-1097. [CrossRef] [PubMed]

14. Wallace, T.M.; Matthews, D.R. The assessment of insulin resistance in man. Diabet. Med. 2002, 19, 527-534. [CrossRef] [PubMed] 
15. Liu, C.S.; Pi-Sunyer, F.X.; Li, C.I.; Davidson, L.E.; Li, T.C.; Chen, W.; Lin, C.C.; Huang, C.Y.; Lin, W.Y. Albuminuria is strongly associated with arterial stiffness, especially in diabetic or hypertensive subjects-a population-based study (Taichung Community Health Study, TCHS). Atherosclerosis 2010, 211, 315-321. [CrossRef] [PubMed]

16. Tomiyama, H.; Yamashina, A.; Arai, T.; Hirose, K.; Koji, Y.; Chikamori, T.; Hori, S.; Yamamoto, Y.; Doba, N.; Hinohara, S. Influences of age and gender on results of noninvasive brachial-ankle pulse wave velocity measurement-A survey of 12517 subjects. Atherosclerosis 2003, 166, 303-309. [CrossRef]

17. Cheng, Y.B.; Li, Y.; Sheng, C.S.; Huang, Q.F.; Wang, J.G. Quantification of the Interrelationship between Brachial-Ankle and Carotid-Femoral Pulse Wave Velocity in a Workplace Population. Pulse 2016, 3, 253-262. [CrossRef]

18. Miyano, I.; Nishinaga, M.; Takata, J.; Shimizu, Y.; Okumiya, K.; Matsubayashi, K.; Ozawa, T.; Sugiura, T.; Yasuda, N.; Doi, Y. Association between brachial-ankle pulse wave velocity and 3-year mortality in community-dwelling older adults. Hypertens. Res. 2010, 33, 678-682. [CrossRef]

19. Suzuki, E.; Kashiwagi, A.; Nishio, Y.; Egawa, K.; Shimizu, S.; Maegawa, H.; Haneda, M.; Yasuda, H.; Morikawa, S.; Inubushi, T.; et al. Increased arterial wall stiffness limits flow volume in the lower extremities in type 2 diabetic patients. Diabetes Care 2001, 24, 2107-2114. [CrossRef]

20. Turin, T.C.; Kita, Y.; Rumana, N.; Takashima, N.; Kadota, A.; Matsui, K.; Sugihara, H.; Morita, Y.; Nakamura, Y.; Miura, K.; et al. Brachial-ankle pulse wave velocity predicts all-cause mortality in the general population: Findings from the Takashima study, Japan. Hypertens. Res. 2010, 33, 922-925. [CrossRef]

21. Aso, K.; Miyata, M.; Kubo, T.; Hashiguchi, H.; Fukudome, M.; Fukushige, E.; Koriyama, N.; Nakazaki, M.; Minagoe, S.; Tei, C. Brachial-ankle pulse wave velocity is useful for evaluation of complications in type 2 diabetic patients. Hypertens. Res. 2003, 26, 807-813. [CrossRef]

22. Chang, L.H.; Lin, H.D.; Kwok, C.F.; Won, J.G.; Chen, H.S.; Chu, C.H.; Hwu, C.M.; Kuo, C.S.; Jap, T.S.; Shih, K.C.; et al. The combination of the ankle brachial index and brachial ankle pulse wave velocity exhibits a superior association with outcomes in diabetic patients. Intern. Med. 2014, 53, 2425-2431. [CrossRef] [PubMed]

23. Katakami, N.; Osonoi, T.; Takahara, M.; Saitou, M.; Matsuoka, T.A.; Yamasaki, Y.; Shimomura, I. Clinical utility of brachial-ankle pulse wave velocity in the prediction of cardiovascular events in diabetic patients. Cardiovasc. Diabetol. 2014, 13, 14-128. [CrossRef] [PubMed]

24. Choi, S.W.; Yun, W.J.; Kim, H.Y.; Lee, Y.H.; Kweon, S.S.; Rhee, J.A.; Choi, J.S.; Shin, M.H. Association between albuminuria, carotid atherosclerosis, arterial stiffness, and peripheral arterial disease in Korean type 2 diabetic patients. Kidney Blood Press Res. 2010, 33, 111-118. [CrossRef] [PubMed]

25. Ishikawa, T.; Hashimoto, J.; Morito, R.H.; Hanazawa, T.; Aikawa, T.; Hara, A.; Shintani, Y.; Metoki, H.; Inoue, R.; Asayama, K.; et al. Association of microalbuminuria with brachial-ankle pulse wave velocity: The Ohasama study. Am. J. Hypertens. 2008, 21, 413-418. [CrossRef] [PubMed]

26. Seo, J.Y.; Kim, M.K.; Choi, B.Y.; Kim, Y.M.; Cho, S.I.; Shin, J. Elevated brachial-ankle pulse wave velocity is independently associated with microalbuminuria in a rural population. J. Korean Med. Sci. 2014, 29, 941-949. [CrossRef] [PubMed]

27. Kawai, T.; Ohishi, M.; Onishi, M.; Ito, N.; Takeya, Y.; Maekawa, Y.; Rakugi, H. Cut-off value of brachial-ankle pulse wave velocity to predict cardiovascular disease in hypertensive patients: A cohort study. J. Atheroscler. Thromb. 2013, 20, 391-400. [CrossRef] [PubMed]

28. Savage, S.; Estacio, R.O.; Jeffers, B.; Schrier, R.W. Urinary albumin excretion as a predictor of diabetic retinopathy, neuropathy, and cardiovascular disease in NIDDM. Diabetes Care 1996, 19, 1243-1248. [CrossRef]

29. Yamashina, A.; Tomiyama, H.; Takeda, K.; Tsuda, H.; Arai, T.; Hirose, K.; Koji, Y.; Hori, S.; Yamamoto, Y. Validity, reproducibility, and clinical significance of noninvasive brachial-ankle pulse wave velocity measurement. Hypertens. Res. 2002, 25, 359-364. [CrossRef]

30. MedCalc Software bvba. MedCalc Software bvba; 17.9.7; MedCalc Software bvba Ostend: Acacialaan, Ostend, Belgium, 2017.

31. Kim, B.J.; Lee, H.A.; Kim, N.H.; Kim, M.W.; Kim, B.S.; Kang, J.H. The association of albuminuria, arterial stiffness, and blood pressure status in nondiabetic, nonhypertensive individuals. J. Hypertens. 2011, 29, 2091-2098. [CrossRef] 
32. Zhao, Q.-h.; Wang, Q.; Zhuang, X.-m.; Wang, P.; Zhang, G.-h. Association between microalbuminuria and brachial-ankle pulse wave velocity in patients with risk factors of cardiovascular disease. Chin. J. Clin. 2012, 6, 570-574.

33. Yokoyama, H.; Hirasawa, K.; Aoki, T.; Ishiyama, M.; Koyama, K. Brachial-ankle pulse wave velocity measured automatically by oscillometric method is elevated in diabetic patients with incipient nephropathy. Diabet Med. 2003, 20, 942-945. [CrossRef] [PubMed]

34. Bellasi, A.; Ferramosca, E.; Ratti, C. Arterial stiffness in chronic kidney disease: The usefulness of a marker of vascular damage. Int. J. Nephrol. 2011, 734832, 23. [CrossRef] [PubMed]

35. Deckert, T.; Feldt-Rasmussen, B.; Borch-Johnsen, K.; Jensen, T.; Kofoed-Enevoldsen, A. Albuminuria reflects widespread vascular damage. The Steno hypothesis. Diabetologia 1989, 32, 219-226. [CrossRef] [PubMed]

36. Dinneen, S.F.; Gerstein, H.C. The association of microalbuminuria and mortality in non-insulin-dependent diabetes mellitus. A systematic overview of the literature. Arch. Intern. Med. 1997, 157, 1413-1418. [CrossRef] [PubMed]

37. Bakris, G.L.; Kuritzky, L. Monitoring and managing urinary albumin excretion: Practical advice for primary care clinicians. Postgrad Med. 2009, 121, 51-60. [CrossRef] [PubMed]

38. McKenna, K.; Thompson, C. Microalbuminuria: A marker to increased renal and cardiovascular risk in diabetes mellitus. Scott. Med. J. 1997, 42, 99-104. [CrossRef] [PubMed]

39. Weir, M.R. Microalbuminuria in type 2 diabetics: An important, overlooked cardiovascular risk factor. J. Clin. Hypertens. 2004, 6, 134-141. [CrossRef]

40. Wang, D.Z.; Tang, Q.; Hua, Q. Prediction of coronary artery disease using pulse wave velocity and retinal artery lesions. Tohoku J. Exp. Med. 2011, 225, 17-22. [CrossRef]

41. Yokoyama, H.; Aoki, T.; Imahori, M.; Kuramitsu, M. Subclinical atherosclerosis is increased in type 2 diabetic patients with microalbuminuria evaluated by intima-media thickness and pulse wave velocity. Kidney Int. 2004, 66, 448-454. [CrossRef]

42. Barbato, A.; D’Elia, L.; Perna, L.; Molisso, A.; Iacone, R.; Strazzullo, P.; Galletti, F. Increased Microalbuminuria Risk in Male Cigarette Smokers: Results from the "Olivetti Heart Study" after 8 Years Follow-Up. Kidney Blood Press Res. 2019, 44, 33-42. [CrossRef]

43. Feodoroff, M.; Harjutsalo, V.; Forsblom, C.; Thorn, L.; Waden, J.; Tolonen, N.; Lithovius, R.; Groop, P.H. Smoking and progression of diabetic nephropathy in patients with type 1 diabetes. Acta Diabetol. 2016, 53, 525-533. [CrossRef] [PubMed]

44. Idowu, A.A.; Ajose, A.O.; Adedeji, A.T.; Adegoke, A.O.; Jimoh, K.A. Microalbuminuria, Other Markers of Nephropathy and Biochemical Derangementsin Type 2 Diabetes Mellitus: Relationships and Determinants. Ghana Med. J. 2017, 51, 56-63. [PubMed]

45. Chatzikyrkou, C.; Menne, J.; Izzo, J.; Viberti, G.; Rabelink, T.; Ruilope, L.M.; Rump, C.; Mertens, P.R.; Haller, H. Predictors for the development of microalbuminuria and interaction with renal function. J. Hypertens. 2017, 35, 2501-2509. [CrossRef] [PubMed]

46. Bjornstad, P.; Maahs, D.M.; Wadwa, R.P.; Pyle, L.; Rewers, M.; Eckel, R.H.; Snell-Bergeon, J.K. Plasma triglycerides predict incident albuminuria and progression of coronary artery calcification in adults with type 1 diabetes: The Coronary Artery Calcification in Type 1 Diabetes Study. J. Clin. Lipidol. 2014, 8, 576-583. [CrossRef] [PubMed]

47. Habib, A.N.; Baird, B.C.; Leypoldt, J.K.; Cheung, A.K.; Goldfarb-Rumyantzev, A.S. The association of lipid levels with mortality in patients on chronic peritoneal dialysis. Nephrol. Dial. Transplant. 2006, 21, 2881-2892. [CrossRef]

48. Lee, M.H.; Kim, H.C.; Ahn, S.V.; Hur, N.W.; Choi, D.P.; Park, C.G.; Suh, I. Prevalence of Dyslipidemia among Korean Adults: Korea National Health and Nutrition Survey 1998-2005. Diabetes Metab J. 2012, 36, 43-55. [CrossRef]

(C) 2019 by the authors. Licensee MDPI, Basel, Switzerland. This article is an open access article distributed under the terms and conditions of the Creative Commons Attribution (CC BY) license (http://creativecommons.org/licenses/by/4.0/). 